\title{
ASYMPTOTIC NONLINEAR WAVE MODELING THROUGH THE DIRICHLET-TO-NEUMANN OPERATOR*
}

\author{
WILLIAM ARTILES ${ }^{\dagger}$ AND ANDRÉ NACHBIN
}

Dedicated to George Papanicolaou with great gratitude

\begin{abstract}
New nonlinear evolution equations are derived that generalize the system by Matsuno [16] and a terrain-following Boussinesq system by Nachbin [23]. The regime considers finite-amplitude surface gravity waves on a two-dimensional incompressible and inviscid fluid of, highly variable, finite depth. The asymptotic simplification of the nonlinear potential theory equations is performed through a perturbation anaylsis of the Dirichlet-to-Neumann operator on a highly corrugated strip. This is achieved through the use of a curvilinear coordinate system. Rather than doing a long wave expansion for the velocity potential, a Fourier-type operator is expanded in a wave steepness parameter. The novelty is that the topography can vary on a broad range of scales. It can also have a complex profile including that of a multiply-valued function. The resulting evolution equations are variable coefficient Boussinesq-type equations. These equations represent a fully dispersive system in the sense that the original (hyperbolic tangent) dispersion relation is not truncated. The formulation is done over a periodically extended domain so that, as an application, it produces efficient Fourier (FFT) solvers. A preliminary communication of this work has been published in the Physical Review Letters [1].
\end{abstract}

Key words. Dispersive waves, inhomogeneous media, asymptotic theory

AMS subject classifications. 76B15, 35Q99, 60F05

1. Introduction. The study of weakly nonlinear (finite-amplitude) water waves has been of great research interest for quite some time. Many fundamental models (namely partial differential equations) have been derived according to the physical situation and carefully analyzed for their mathematical properties. In a recent paper Matsuno [16] derived a Boussinesq-type model that arises from an expansion in a steepness parameter and not in the dispersion parameter corresponding to a long wave regime. Matsuno develops a method based on the theory of complex functions and a systematic perturbation theory with respect to the steepness parameter $\varepsilon \equiv a / \ell$ ( $a$ is the amplitude scale and $\ell$ is a wavelength scale). We start by using dimensionless parameters such as in Whitham's book [30] and in [23]: let $\alpha=a / h_{o}$ be the nonlinearity parameter (with the typical, say average depth denoted by $h_{o}$ ) and $\beta=h_{o}^{2} / \ell^{2}$ be the dispersion parameter. As pointed out in [16] the formulation naturally suggests combining these parameters as $\alpha \sqrt{\beta} \equiv \varepsilon$. Hence the characteristic depth $h_{o}$ cancels out and the free surface perturbations are controlled through $\varepsilon$. The starting point for the asymptotic analysis are the dimensionless potential theory equations.

The novelty in this paper is that we accommodate the asymptotic modeling to consider very general topographic profiles, as for example the one presented in Fig. 1.1. We also start from the nonlinear, dimensionless potential theory equations but follow an analytical route that is somewhat different from Matsuno's. Naturally some of the transforms used are similar, but they are "brought into the picture" by different means. In particular the Fourier analysis presented naturally suggests the use of FFT based methods to generate efficient numerical schemes. The formulation presented

\footnotetext{
${ }^{*}$ Received September 14, 2004; accepted for publication January 7, 2005. This work was supported by CNPq/Brazil under Grant 300368/96-8.

†Instituto de Matemática Pura e Aplicada, Est. D. Castorina 110, Jardim Botânico, Rio de Janeiro, RJ 22460-320, Brazil (william@impa.br; nachbin@impa.br).
} 


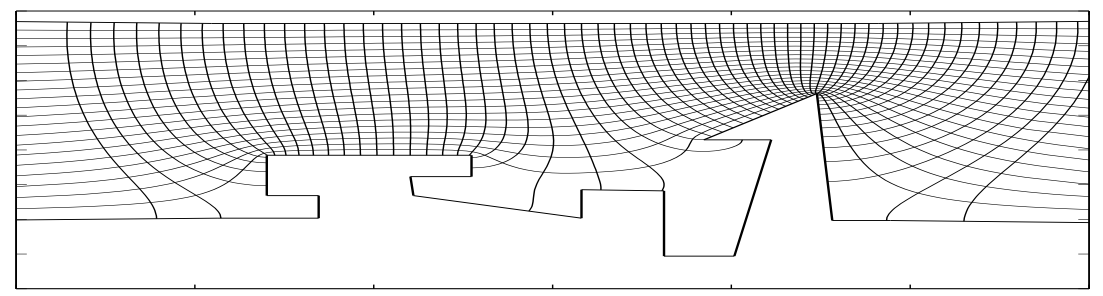

FIG. 1.1. Topography profile together with the level curves of the $\xi \zeta$ coordinate system.

here also extends a, recently published [23], weakly dispersive terrain-following Boussinesq system. A regime of interest is pulse shaped surface gravity waves interacting with rapidly varying, disordered, topographies.

We use an orthogonal curvilinear coordinate system for the potential theory equations. Within this frame we are able to write a Dirichlet-to-Neumann $(D t N)$ operator which automatically reduces the entire dynamics to the free surface. This formulation is possible in the presence of complex multi-valued profiles, or even rapidly varying topographies. Our transforms not only resemble, but are clearly related to, those in Matsuno [16] and in Byatt-Smith [4]. Some of the differences are that we work in Fourier space while Matsuno uses complex functions. We define our curvilinear coordinate system through the conformal mapping of the undisturbed corrugated channel, while Byatt-Smith maps the (finite-amplitude) time dependent flat channel (i.e. without a topography). As mentioned by Matsuno [16] the equations presented in [4] are quite complicated. In our formulation we work in the physical domain, through the curvilinear coordinate system and we arrive at Fourier-type transforms which are easily incorporated into a numerical method. The final result is a, variable coefficient, weakly nonlinear evolution equation of the Boussinesq-type. Nonlinear waves in a highly heterogeneous medium is of great interest in Physics and Mathematics. Boussinesq-type models are of great interest in Geophysical applications. Recently, using a power series expansion for the velocity potential, Nachbin [23] derived a terrainfollowing Boussinesq system valid for weakly dispersive, weakly nonlinear waves in the presence of a rapidly varying topography. This model was used to study long waves interacting with disordered (randomly varying) topographies [17, 18, 10, 12]. In particular for the time-reversal refocusing of solitary waves $[18,12]$. Our generalization of Matsuno's Boussinesq-type system has, as its (weakly dispersive) leading order approximation the terrain-following system presented in Nachbin [23]. This is a nice consistency check since here we do not use a power series expansion for the potential, but rather we do asymptotics with the Fourier operators.

Other very recent work include that of Craig and Sulem [5] which formulated an efficient spectral method based on the $D t N$ operator. Their analysis differs from ours and Matsuno's but some resulting transforms are similar, in particular the Hilbert transform on a strip: namely equation (4.4) in the present paper, which appears as (18) in Matsuno [16] and as (27) in Craig and Sulem [5]. We recall that Matsuno's [16] as well as Craig and Sulem's analysis [5] are restricted to flat bottoms. Along these lines Zakharov et al. [31] using a Hamiltonian structure deduced exact (nonlinear) free surface equations in a fluid of infinite depth. The free surface is conformally mapped and Hilbert-differential equations are obtained along the free surface. The presence of Hilbert transforms has a clear connection with the three strategies mentioned above (including the present one). This is explicitly mentioned by Zakharov 
et al. [31] in a reference to the work of Craig and Sulem [5]. In the presence of highly variable topographies we point out the very recent work by Keller [15]. A shallow water (hyperbolic) model is derived from the Euler equations by using curvilinear coordinates. This curvilinear coordinate system is based on smooth topographies or a smooth curve near the topography. We recall that in the present work our curvilinear coordinate system arises from a conformal transformation and therefore is valid for polygonal bottom profiles which need not be single-valued. Moreover the resulting dispersive system can be reduced to a shallow water (hyperbolic) system by only keeping $O(\sqrt{\beta})$ terms (in the metric term $M(\xi)$ to be defined in the next section). This has been discussed in more detail in Nachbin [23].

This paper is organized as follows. In section 2 we present a brief introduction to the free boundary problem formulated through the nonlinear potential theory. In section 3 we recall Guidotti's (time independent) formulation of the Dirichlet-toNeumann operator on a corrugated halfplane and apply it to a corrugated strip. Its time dependent extension for nonlinear gravity waves is done in section 4 where a new (variable coefficient) Boussinesq-type system is derived. In section 5 we briefly outline a numerical application of the $D t N$ operator. Namely we present a very efficient spectral method for the linear potential theory problem. The complete nonlinear version, together with numerical experiments for rapidly varying topographies, will be presented elsewhere. As mentioned above recently Craig and Sulem [5] and Zakharov et al. [31] formulated efficient spectral methods based on the DtN transformations. Their methods does not account for the presence of a topography.

A preliminary communication of this work has been published in the Physical Review Letters [1].

2. The inviscid and incompressible free boundary problem. Following Whitham [30] and Rosales and Papanicolaou [27] the dimensionless nonlinear potential theory equations are

$$
\beta \phi_{x x}+\phi_{y y}=0, \quad \text { in } \quad \Omega,
$$

with free surface conditions

$$
\left\{\begin{array}{ll}
\phi_{t}+\frac{\alpha}{2}\left(\phi_{x}^{2}+\frac{1}{\beta} \phi_{y}^{2}\right)+\eta & =0 \\
\eta_{t}+\alpha \phi_{x} \eta_{x}-\frac{1}{\beta} \phi_{y} & =0
\end{array} \quad \text { at } \quad y=\alpha \eta(x, t)\right.
$$

and a Neumann condition

$$
\frac{\beta}{\gamma} h^{\prime}\left(\frac{x}{\gamma}\right) \phi_{x}+\phi_{y}=0 \quad \text { along } \quad y=-\sqrt{\beta} h\left(\frac{x}{\gamma}\right),
$$

the highly variable topography, where

$$
\Omega=\left\{(x, y) \in \mathbb{R}^{2}, \quad \text { and } \quad-\sqrt{\beta} h\left(\frac{x}{\gamma}\right)<y<\alpha \eta(x, t)\right\} .
$$

Having defined the dimensionless parameters $\alpha$ and $\beta$, we are also led to defining a parameter controlling the depth irregularities: $\gamma \equiv \ell_{b} / \ell$, where $\ell_{b}$ is the (horizontal) characteristic length scale for the bottom variations, described through $y=-\sqrt{\beta} h(x / \gamma)$ [23]. The topography's amplitude does not need to be small. In other words, in general $h(x / \gamma)=1+n(x / \gamma)$ where $n$ does not need to be small. The $\sqrt{\beta}$ multiplying $h(x / \gamma)$ is due to the shallow water scaling. Hence the average depth is small and equal to $\sqrt{\beta}$. If $\gamma \ll 1$ the topography is rapidly varying. Using the Schwarz-Christoffel transformation $[7,8]$ we define a mapping from a uniform strip in 


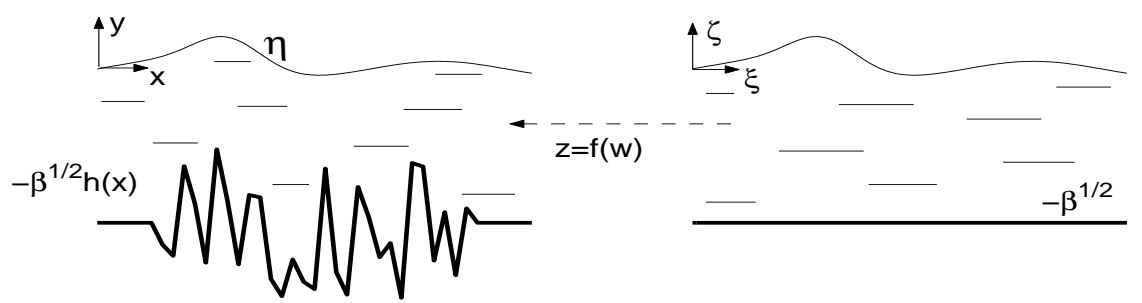

FIG. 2.1. Setup for the Schwarz-Christoffel transformation where $z=x+i y$ and $w=\xi+i \zeta$. The mapping is defined for the undisturbed configuration (i.e. with $\eta \equiv 0$ ).

the $\xi \zeta$-plane onto the undisturbed $(\eta \equiv 0)$ corrugated strip in the physical $x y$-plane (c.f. Fig. 2.1). This is possible even when $h(x / \gamma)$ represents a polygonal, multi-valued profile as in Fig. 1.1. This formulation defines $x(\xi, \zeta)$ and $y(\xi, \zeta)$ as harmonic functions together with $(\xi, \zeta)$ level curves of an orthogonal curvilinear coordinate system in the physical domain (c.f. Fig 1.1). In the curvilinear coordinate system we have that $\left(\partial_{\xi \xi}+\partial_{\zeta \zeta}\right)=|J|^{2}\left(\partial_{x x}+\partial_{y y}\right)$, where by the Cauchy-Riemann equations [23], the Jacobian of the $(\xi, \zeta) \mapsto(x, y)$ transformation is $|J| \equiv y_{\xi}^{2}+y_{\zeta}^{2}$. We point out that in [23] one works with a unit depth, rather than $\sqrt{\beta}$. Hence $(\xi, \zeta)$ denotes a coordinate system slightly different from that in [23]. Initially, for convenience, we will keep track of the free surface (FS) elevation in both coordinate systems. In the mapped domain we have $\zeta \equiv S(\xi, t)=\varepsilon N(\xi, t)$, while in the physical domain it is defined by $y(\xi, S(\xi, t))=\alpha \eta(x(\xi, S(\xi, t)), t)$, with $\eta$ denoting the surface gravity wave. When the FS has a small steepness (as indicated by $\varepsilon N$ above) the Jacobian can be approximated as $|J| \approx y_{\zeta}^{2}(\xi, 0)+O\left(\varepsilon^{2}\right)[23]$ and the relevant FS metric coefficient is $M(\xi ; \sqrt{\beta}, \gamma) \equiv y_{\zeta}(\xi, 0)$, where

$$
M(\xi ; \sqrt{\beta}, \gamma)=\frac{\pi}{4 \sqrt{\beta}} \int_{-\infty}^{\infty} \frac{h\left(x\left(\xi_{o},-\sqrt{\beta}\right) / \gamma\right)}{\cosh ^{2} \frac{\pi}{2 \sqrt{\beta}}\left(\xi_{o}-\xi\right)} d \xi_{o}
$$

The metric term carries vertical and horizontal bottom variation scales through the parameters $\sqrt{\beta}$ and $\gamma$.

Laplace's equation, for the velocity potential $\phi$, is invariant through our change of coordinates. Thus the nonlinear potential theory equations in curvilinear $\xi \zeta$ coordinates, as presented in Nachbin [23] are

$$
\phi_{\xi \xi}+\phi_{\zeta \zeta}=0, \quad-\sqrt{\beta}<\zeta<S(\xi, t) .
$$

where at the FS the free boundary conditions are

$$
\begin{gathered}
N_{t}+\frac{\alpha}{|J|} \phi_{\xi} N_{\xi}-\frac{1}{|J| \sqrt{\beta}} \phi_{\zeta}=0 \\
\phi_{t}+\frac{\alpha}{2|J|}\left(\phi_{\xi}^{2}+\phi_{\zeta}^{2}\right)+\eta=0 .
\end{gathered}
$$

We have a trivial Neumann condition along the impermeable topography $\zeta \equiv-\sqrt{\beta}$ : $\phi_{\zeta}=0$. No approximation has been made up to this point.

To obtain a terrain-following (weakly dispersive, weakly nonlinear) Boussinesq system, Nachbin [23] considered a power series expansion near the bottom of the 
channel (translated to be at $\zeta=0$ ) in the form

$$
\phi(\xi, \zeta, t)=\sum_{n=0}^{\infty} \zeta^{n} f_{n}(\xi, t) .
$$

This function satisfies the scaled Laplace equation when

$$
f_{m+2}=\frac{-\beta}{(m+2)(m+1)} \frac{\partial^{2} f_{m}}{\partial \xi^{2}} .
$$

Moreover the Neumann condition at the bottom is satisfied when all odd terms (i.e. $\left.f_{2 m+1}\right)$ are zero. Hence by denoting $f_{0}(\xi, t)=f(\xi, t)$ for simplicity, we have the velocity potential

$$
\phi(\xi, \zeta, t)=\sum_{n=0}^{\infty} \frac{(-\beta)^{n}}{(2 n) !} \zeta^{2 n} \frac{\partial^{2 n} f(\xi, t)}{\partial \xi^{2 n}}
$$

as a power series expansion in $\beta$. Then this series is substituted in the nonlinear free surface conditions and the leading order terms in $\alpha$ and $\beta$ are retained. As soon as the power series is truncated we have an approximate potential. Correspondingly the linear dispersion relation

$$
\omega^{2}=\frac{k}{\sqrt{\beta}} \tanh (k \sqrt{\beta})
$$

will also be truncated. This strategy is exactly the same as in Whitham's book [30] for a flat channel in cartesian coordinates. We will show that by working with the DtN operator we will be able to keep the full dispersion relation, for the underlying linear system.

In the sequel we will calculate the source distribution for the integral representation of the FS's Dirichlet data and its impact on representing the $D t N$ operator. For a nonlinear FS the source distribution is found implicitly and through a mild steepness hypothesis $(\varepsilon \ll 1)$ we find an efficient approximation for the $D t N$ operator.

3. The Dirichlet-to-Neumann $(D t N)$ operator. In this section we start with a brief review on Guidotti's time independent strategy [13] to formulate a DtN operator for a halfplane configuration as in Fig. 3.1. Then we will adapt this strategy to a time independent problem for a corrugated strip. Once this has been achieved we will, in a following section, migrate this formulism to the nonlinear wave evolution problem and reduce the entire dynamics to the free surface.

3.1. The Dirichlet problem on a halfplane. In this subsection, following [13], attention is given to periodic problems (say of period one) such that

$$
\varphi(\xi), S(\xi) \in C_{p e r}([0,1]) .
$$

The notation is such that Guidotti considers Laplace's equation in the domain $\Omega$, above the curve $\Gamma \equiv(\xi, S(\xi))$, with Dirichlet data $\varphi(\xi)$ imposed along $\Gamma$. By using periodicity we are preparing our asymptotic models for FFT based numerical schemes. Obviously there is no loss of generality when we normalize the period. Let $(\xi, S(\xi))$ be a parametrization of the halfplane's (lower) boundary curve $\Gamma$. We adopt the 


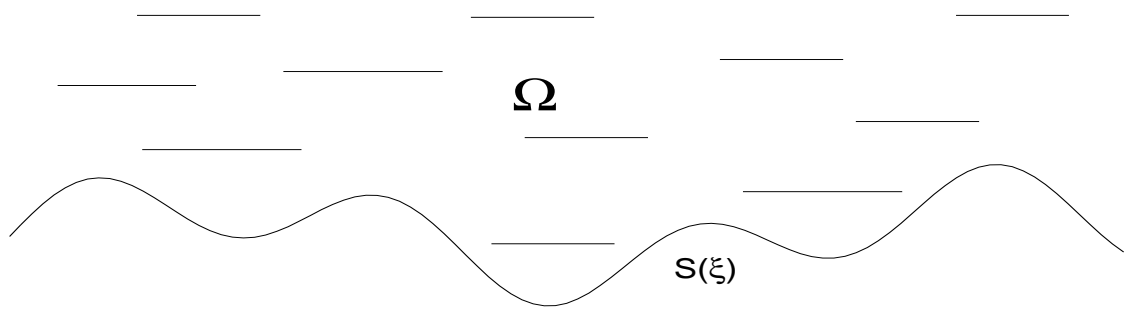

FIG. 3.1. Upper halfplane bounded by a corrugated curve $S(\xi)$.

following notation along the boundary: the mapping that takes the Dirichlet data onto its corresponding Neumann data we denote as

$$
\operatorname{DtN}[\varphi](\xi)=\partial_{n} \phi(\xi, S(\xi)),
$$

where

$$
\partial_{n}:=\vec{n} \cdot\left(\partial_{\xi}, \partial_{\zeta}\right), \quad \vec{n}=\left|\Gamma_{\xi}\right|^{-1}\left(-S_{\xi}(\xi), 1\right) \quad \text { and } \quad\left|\Gamma_{\xi}\right|=\sqrt{1+S_{\xi}^{2}(\xi)} .
$$

As in classical potential theory, Guidotti suggests an integral representation for the potential, in the form

$$
\phi(w)=\int_{\Gamma} G(w-\tilde{w}) f(\tilde{w}) d \Gamma, \quad \tilde{w} \in \Gamma .
$$

The unknown function $f$ will be determined by the potential's Dirichlet data along $\Gamma$. The kernel is chosen so that this representation satisfies Laplace's equation and also so that FFTs are applicable. This will lead to efficient numerical schemes with exact dispersive properties for a wide class of initial data. As mentioned in the Introduction, recently this has been exploited numerically by other authors [5, 31]. By $d \Gamma$ we represent an infinitesimal boundary element and by $w=(\xi, \zeta)$ an interior point of our domain $\Omega$. The 1-periodic version of the integral representation (3.2) is

$$
\phi(\xi, \zeta)=\int_{0}^{1} G(\xi-\tilde{\xi}, \zeta-S(\tilde{\xi})) f(\tilde{\xi})\left|\Gamma_{\tilde{\xi}}\right| d \tilde{\xi} .
$$

The periodic kernel proposed by Guidotti [13] has logarithmic singularities, in the form

$$
G(\xi, \zeta)=\frac{1}{2 \pi} \ln \left(1+e^{-4 \pi \zeta}-2 e^{-2 \pi \zeta} \cos (2 \pi \xi)\right),
$$

and can also be represented by the Fourier series

$$
G(\xi, \zeta)=-\sum_{\kappa \neq 0} \frac{1}{2 \pi|\kappa|} e^{-2 \pi|\kappa| \zeta} e^{2 \pi i \kappa \xi} \quad \text { if } \quad \zeta \geq 0 .
$$

Hence the kernel is a harmonic function in the halfplane $\xi, \zeta>0$, and 1-periodic in its first variable with the property that [13]

$$
\lim _{\zeta \rightarrow 0^{+}} \partial_{\zeta} G(\xi, \zeta)=\lim _{\zeta \rightarrow 0^{+}} \frac{-2 e^{-4 \pi \zeta}+2 e^{-2 \pi \zeta} \cos (2 \pi \xi)}{1+e^{-4 \pi \zeta}-2 e^{-2 \pi \zeta} \cos (2 \pi \xi)}=\delta(\xi)-1 .
$$


The Dirac delta is denoted by $\delta(\xi)$. Therefore one needs to be careful when deducing the $D t N$ operator since an interior point will approach a boundary curve. This result is summarized in the following lemma proved in [13]:

Lemma. Let $G$ be the Green's functions given above. Suppose that $S(\xi) \in$ $C_{\text {per }}^{1}([0,1])$ represents a corrugated curve along the boundary of $\Omega$. Then the following limit holds as an interior point approaches this boundary:

$$
\begin{gathered}
\lim _{w \rightarrow w_{o}} \int_{\Gamma} \partial_{n(w)} G(w-\tilde{w}) f(\tilde{w}) d \Gamma_{\tilde{w}}=-f\left(w_{o}\right)+f_{\Gamma} \partial_{n\left(w_{o}\right)} G\left(w_{o}-\tilde{w}\right) f(\tilde{w}) d \Gamma_{\tilde{w}} \\
\text { when } \quad w \rightarrow w_{o}=\left(\xi_{o}, S\left(\xi_{o}\right)\right) \in \Gamma .
\end{gathered}
$$

The limiting integral is given as a Cauchy Principal Value (indicated by a dash). The interior point can not approach the boundary curve in the tangential direction. The dummy variable of integration is indicated in the element $d \Gamma_{\tilde{w}}$. obtain

Clearly from the potential's integral representation (3.2) along the boundary we

$$
\varphi\left(w_{o}\right)=\int_{\Gamma} G\left(w_{o}-\tilde{w}\right) f(\tilde{w}) d \Gamma_{\tilde{w}}
$$

where $w_{o}$ is also along the boundary. Its periodic counterpart leads to the following Fredholm equation of the first kind in the source density $f$ :

$$
\varphi(\xi)=\int_{0}^{1} G(\xi-\tilde{\xi}, \zeta-S(\tilde{\xi})) f(\tilde{\xi})\left|\Gamma_{\tilde{\xi}}\right| d \tilde{\xi} .
$$

Technical details on integral equations can be found in Taylor [29].

Once we find the singularity density $f(\xi)$ we use (3.2) (or its periodic counterpart (3.3)) and the lemma above to deduce an integral representation for the Dirichlet-toNeumann operator $\operatorname{DtN}(\phi)=\partial_{n} \phi$ :

$$
\operatorname{DtN}(\varphi)\left(w_{o}\right)=\lim _{w \rightarrow w_{o}} \int_{\Gamma} \partial_{n(w)} G(w-\tilde{w}) f(\tilde{w}) d \Gamma_{\tilde{w}}
$$

where $w_{o} \in \Gamma$. In the periodic regime we have

$$
D t N(\varphi)\left(\xi_{o}\right)=-f\left(\xi_{o}\right)+f_{0}^{1} \partial_{n\left(\xi_{o}\right)} G\left(\xi_{o}-\tilde{\xi}, S\left(\xi_{o}\right)-S(\tilde{\xi})\right) f(\tilde{\xi})\left|\Gamma_{\tilde{\xi}}\right| d \tilde{\xi} .
$$

Formally this completes the calculation of the $D t N$ operator, considering that at this stage the density $f$ is known.

Summarizing what we have presented up to this point: in order to find the (consistent) Neumann condition for the periodic Dirichlet problem we use the integral representation (3.3) for the potential. Then

1. we find $f(\xi)$, the logarithmic singularity's density distribution, by inverting the integral equation (3.7), with the kernel (3.4) and

2. we evaluate equation (3.8) using the corresponding expression for $f(\xi)$.

Recall that the Dirichlet data was denoted as $\phi(\xi, S(\xi))=\varphi(\xi) \equiv \varphi(w)$ according to (3.6). In the next subsection we will see how to invert expression (3.7) for $f(\xi)$ in an exact fashion for the linear problem, and approximately in the weakly nonlinear regime. 


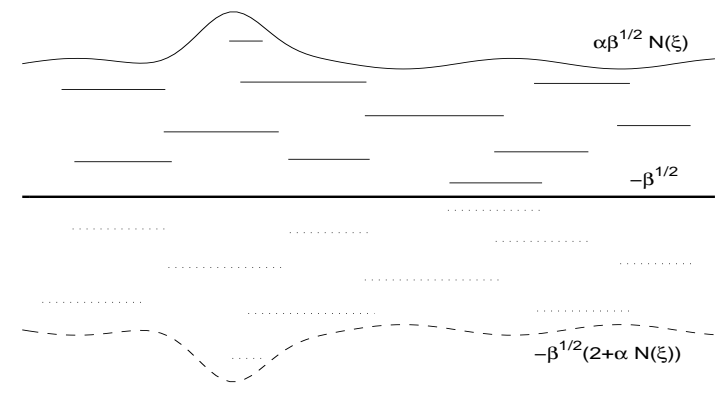

FIG. 3.2. Reflected $\xi \zeta$-domain in order to give rise to a Dirichlet problem. The FS, in the water wave problem, is described by $S(\xi)=\varepsilon N(\xi) ; \varepsilon \equiv \alpha \sqrt{\beta}$. The dashed curve is the FS's reflected image.

3.2. The Dirichlet problem on a corrugated strip. We now prepare the formulation of the $D t N$ operator for the finite depth wave problem based on Guidotti's [13] work (for a time independent harmonic function on a corrugated half-plane) as was presented above. To simplify the presentation we first analyze the harmonic problem at a frozen instant of time:

$$
\begin{aligned}
\phi_{\xi \xi}+\phi_{\zeta \zeta} & =0 \text { in }-\sqrt{\beta}<\zeta<S(\xi) \\
\phi(\xi, S(\xi)) & =\varphi(\xi) \text { at the FS } \zeta=S(\xi) \\
\phi_{\zeta}(\xi,-\sqrt{\beta}) & =0 \text { at the topography } \zeta=-\sqrt{\beta} .
\end{aligned}
$$

Our goal is to express the Neumann data directly from its corresponding Dirichlet data $\varphi(\xi)$. By reflecting our domain about the topographic level curve $\zeta \equiv-\sqrt{\beta}$ (as in Fig. 3.2) we convert the problem above into the Dirichlet problem

$$
\begin{aligned}
\phi_{\xi \xi}+\phi_{\zeta \zeta} & =0 \text { in } \quad-2 \sqrt{\beta}-S(\xi)<\zeta<S(\xi) \\
\phi & =\varphi(\xi) \text { at } \zeta=S(\xi) \\
\phi & =\varphi(\xi) \text { at } \zeta=-S(\xi)-2 \sqrt{\beta} .
\end{aligned}
$$

Moreover, due to the symmetry about $\zeta \equiv-\sqrt{\beta}$ we have that $\phi_{\zeta}(\xi,-\sqrt{\beta})=0$ is automatically satisfied. We proceed in obtaining an integral representation for the solution of problem (3.12)-(3.14). We again extend our domain periodically and normalize it to having period one. The time independent potential is cast into the integral representation

$$
\phi(\xi, \zeta)=\int_{0}^{1} K(\xi, \tilde{\xi}, \zeta, S(\tilde{\xi})) f(\tilde{\xi})\left|\Gamma_{\tilde{\xi}}\right| d \tilde{\xi},
$$

where now the kernel has the form $K(\xi, \tilde{\xi}, \zeta, S(\tilde{\xi}))=G(s,-\zeta+\tilde{S})+G(s, \zeta+\tilde{S}+2 \sqrt{\beta})$. Our notation is such that $f(\tilde{\xi})$ is the symmetric source distribution over the top and bottom boundary curves, $\left|\Gamma_{\xi}\right|^{2} \equiv 1+S_{\xi}^{2}(\xi), s=\xi-\tilde{\xi}$ and $\tilde{S}=S(\tilde{\xi})$. One can then verify that the integral representation satisfies $\phi_{\zeta}(\xi,-\sqrt{\beta})=0$ and $\phi(\xi, \zeta)=$ $\phi(\xi,-\zeta-2 \sqrt{\beta})$ as expected. Thus we have formally solved the potential problem (3.9)-(3.11) once we find the source distribution $f(\xi)$.

Before presenting an approximate strategy for finding the source distribution $f(\xi)$ we recall the setup for defining the $D t N$ operator. The integral representation 
in $(3.15)$ can be written as

$$
\begin{array}{r}
\phi(\xi, \zeta)=\int_{0}^{1} K(\xi, \tilde{\xi}, 0,0) f(\tilde{\xi})\left|\Gamma_{\tilde{\xi}}\right| d \tilde{\xi}+ \\
+\int_{0}^{1}\{K(\xi, \tilde{\xi}, \zeta, S(\tilde{\xi}))-K(\xi, \tilde{\xi}, 0,0)\} f(\tilde{\xi})\left|\Gamma_{\tilde{\xi}}\right| d \tilde{\xi} .
\end{array}
$$

The first term, containing $K(\xi, \tilde{\xi}, 0,0)$, is the linear (singular) term corresponding to infinitesimal perturbations about the undisturbed water surface. In this way the nonlinear term arises as a desingularized correction term. Taking the normal derivative of the potential in (3.16) and letting the interior point approach the top boundary we obtain, in the limit, the Dirichlet-to-Neumann operator $\operatorname{Dt} N(\varphi)(\xi) \equiv \partial \phi / \partial n(\xi, S(\xi))$, which gives the Neumann data along the FS.

Now we go back to calculating the source distribution $f(\xi)$ along the FS. Motivated by (3.16) we decompose the Dirichlet data into its linear and nonlinear parts: $\varphi(\xi) \equiv \varphi_{L}(\xi)+\varphi_{N L}(\xi)$.

The linear contribution. Using the Fourier representation of the Green's function $G$ in the linear term of (3.16), and carefully computing the limit as we approach the top boundary, we have that $\varphi_{L}(\xi)=\mathbf{P}\left[f\left|\Gamma_{\xi}\right|\right]$ where

$$
\mathbf{P}\left[f\left|\Gamma_{\xi}\right|\right] \equiv-\sum_{\kappa \neq 0} \frac{1+e^{-2 \pi|\kappa| 2 \sqrt{\beta}}}{2 \pi|\kappa|} \mathbf{F}_{\kappa}\left[f\left|\Gamma_{\xi}\right|\right] e^{2 \pi i \kappa \xi} .
$$

By $\mathbf{F}_{\kappa}[g]$ we denote the k-th Fourier coefficient of the one-periodic function $g(\xi)$. The contribution from the linear part is easily computed through $f\left|\Gamma_{\xi}\right|=\mathbf{P}^{-1}\left[\varphi_{L}\right]$. This is equivalent to the (linear) flat FS case, but recall that for weakly nonlinear waves $S(\xi)=O(\varepsilon)$. Hence we will seek an $O(\varepsilon)$ approximation of $f(\xi)$ from the full representation (3.16).

The nonlinear contribution. The integral equation (3.16), evaluated along the boundary curve $\zeta=S(\xi)$, can be written in a more compact (operator) notation

$$
\varphi(\xi)=\mathbf{P}\left[f\left|\Gamma_{\xi}\right|\right]+\mathbf{Q}_{S}\left[f\left|\Gamma_{\xi}\right|\right] .
$$

The nonlinear ( $S$-dependent) contribution is identified by the operator $\mathbf{Q}_{S}[\cdot]$. Applying $\mathbf{P}^{-1}$ at both sides and assuming that $\left(\mathbf{I}+\mathbf{P}^{-1} \mathbf{Q}_{S}\right)$ has an inverse, we formally arrive at

$$
f\left|\Gamma_{\xi}\right|=\left(\mathbf{I}+\mathbf{P}^{-1} \mathbf{Q}_{S}\right)^{-1} \mathbf{P}^{-1}[\varphi],
$$

where $\mathbf{I}$ denotes the identity. Existence conditions for the solution of equations as (3.18) are discussed in [29]. For example let $\varphi$ be continuous with period one. We observe that along the top boundary $S(\xi)$ the second part of the kernel $K$, namely $G(\xi-\tilde{\xi}, \zeta+\tilde{S}+2 \sqrt{\beta})$, is continuous and therefore the lemma above applies in the exact same way as before.

The main difficulty resides on inverting (3.16) and computing the source distribution $f(\xi)$ along the nonlinear FS, in the presence of a kernel depending on $S$. This was formally indicated in (3.19) but in reality we will perform an $O(\varepsilon)$ approximation in (3.18) so that $\phi(\xi, S)=\varphi(\xi) \approx \mathbf{P}\left[f\left|\Gamma_{\xi}\right|\right]+\mathbf{R}_{S}\left[f\left|\Gamma_{\xi}\right|\right]$. As will be shown below, the operator $\mathbf{R}_{S}$, which still depends on the FS profile $S$, is an $O(\varepsilon)$ approximation to the full nonlinear operator $\mathbf{Q}_{S}$. 
From the definition of the Green's function we have that

$$
\begin{aligned}
& \mathbf{Q}_{S}\left[f\left|\Gamma_{\xi}\right|\right]=\frac{1}{2 \pi} \int_{0}^{1} \ln \frac{1+e^{-4 \pi(-S+\tilde{S})}-2 e^{2 \pi(-S+\tilde{S})} \cos [2 \pi(\xi-\tilde{\xi})]}{2-2 \cos [2 \pi(\xi-\tilde{\xi})]} f \Gamma_{\tilde{\xi}} \mid d \tilde{\xi}+ \\
& +\frac{1}{2 \pi} \int_{0}^{1} \ln \frac{1+e^{-4 \pi(S+\tilde{S}+2 \sqrt{\beta})}-2 e^{-2 \pi(S+\tilde{S}+2 \sqrt{\beta})} \cos [2 \pi(\xi-\tilde{\xi})]}{1+e^{-4 \pi 2 \sqrt{\beta}}-2 e^{-2 \pi 2 \sqrt{\beta}} \cos [2 \pi(\xi-\tilde{\xi})]} f(\tilde{\xi})\left|\Gamma_{\tilde{\xi}}\right| d \tilde{\xi}
\end{aligned}
$$

where $S=O(\varepsilon)$. In the neighborhood of the boundary curve $S$ we take a generic interior point $(\xi-a, S(\xi)-b) \in \Omega$ (with $\left.r_{\varepsilon_{\tilde{z}}}^{2} \equiv a^{2}+b^{2}\right)$ and we perform an expansion in $\varepsilon$ for the desingularized kernel $K(\xi-a, \tilde{\xi}, S-b, \tilde{S})-K(\xi, \tilde{\xi}, 0,0)$ of the nonlinear contribution. But we do this with the corresponding Fourier representation of the kernel. Keeping the first term we have that

$$
G(\xi-a-\tilde{\xi},-S+b+\tilde{S})-G(\xi-a-\tilde{\xi}, b) \approx-(S-\tilde{S}) \sum_{\kappa \neq 0} e^{-2 \pi|\kappa| b} e^{2 \pi i \kappa(\xi-a-\tilde{\xi})}+\ldots
$$

On the other hand the kernel's behavior along the lower boundary of integration (namely $K(\xi-a, \tilde{\xi}, S-b, \tilde{S})-K(\xi, \tilde{\xi}, 0,0)$ ) presents no difficulty and

$$
G(\xi-\tilde{\xi}, S+\tilde{S}+2 \sqrt{\beta})-G(\xi-\tilde{\xi}, 2 \sqrt{\beta}) \approx(S+\tilde{S}) \sum_{\kappa \neq 0} e^{-2 \pi|\kappa| 2 \sqrt{\beta}} e^{2 \pi i \kappa(\xi-\tilde{\xi})}+\ldots
$$

for its series representation converges absolutely even when $b=0$.

As a result, to leading order, $\mathbf{Q}_{S}$ has a series representation

$$
\begin{aligned}
\mathbf{Q}_{S}\left[f\left|\Gamma_{\xi}\right|\right]=-\lim _{r_{\varepsilon} \rightarrow 0^{+}} & \int_{0}^{1}(S-\tilde{S}) \sum_{\kappa \neq 0} e^{-2 \pi|\kappa| b} e^{2 \pi i \kappa(\xi-a-\tilde{\xi})} f(\tilde{\xi})\left|\Gamma_{\tilde{\xi}}\right| d \tilde{\xi}+ \\
& +\int_{0}^{1}(S+\tilde{S}) \sum_{\kappa \neq 0} e^{-2 \pi|\kappa| 2 \sqrt{\beta}} e^{2 \pi i \kappa(\xi-\tilde{\xi})} f(\tilde{\xi})\left|\Gamma_{\tilde{\xi}}\right| d \tilde{\xi}+O\left(\varepsilon^{2}\right) .
\end{aligned}
$$

The dominant series expansions converge absolutely and we exchange summation with integration. By taking the limit we characterize the leading order approximation $\mathbf{R}_{S}$ to the fully nonlinear operator $\mathbf{Q}_{S}$. Namely by rearranging terms we have that

$$
\begin{aligned}
\mathbf{R}_{S}\left[f\left|\Gamma_{\xi}\right|\right]=S \sum_{\kappa \neq 0}\left\{-1+e^{-2 \pi|\kappa| 2 \sqrt{\beta}}\right\} \mathbf{F}_{\kappa}[ & \left.f\left|\Gamma_{\xi}\right|\right] e^{2 \pi i \kappa \xi}+ \\
& +\sum_{\kappa \neq 0}\left\{1+e^{-2 \pi|\kappa| 2 \sqrt{\beta}}\right\} \mathbf{F}_{\kappa}\left[S f\left|\Gamma_{\xi}\right|\right] e^{2 \pi i \kappa \xi} .
\end{aligned}
$$

By using the approximate operator $\mathbf{P}^{-\mathbf{1}}\left(\mathbf{I}-\mathbf{R}_{\mathbf{S}} \mathbf{P}^{-\mathbf{1}}\right)$, to leading order, we obtain

$$
f(\xi)\left|\Gamma_{\xi}\right|=\mathbf{P}^{-1}[\varphi]-\mathbf{P}^{-1} \mathbf{R}_{S} \mathbf{P}^{-1}[\varphi]+O\left(\varepsilon^{2}\right) .
$$

Using the Fourier representation of $\mathbf{R}_{S}$, and the inversion formula as we did above for the linear analysis, we arrive at

$$
\begin{array}{r}
\mathbf{R}_{S} \mathbf{P}^{-1}[\varphi]=S \sum_{\kappa \neq 0} 2 \pi \kappa \tanh (2 \pi \kappa \sqrt{\beta}) \mathbf{F}_{\kappa}[\varphi] e^{2 \pi i \kappa \xi}+ \\
+\sum_{\kappa \neq 0}\left\{1+e^{-2 \pi|\kappa| 2 \sqrt{\beta}}\right\} \mathbf{F}_{\kappa}\left[S \mathbf{P}^{-1}[\varphi]\right] e^{2 \pi i \kappa \xi} .
\end{array}
$$


Then for the approximate (weakly nonlinear) inversion procedure (3.20), we have that

$$
f(\xi)\left|\Gamma_{\xi}\right|=\mathbf{P}^{-1}[\varphi]-\mathbf{P}^{-1}\left[S \operatorname{Dt} N_{0}[\varphi]\right]+\sum_{\kappa \neq 0} 2 \pi|\kappa| \mathbf{F}_{\kappa}\left[S \mathbf{P}^{-1}[\varphi]\right] e^{2 \pi i \kappa \xi}+O\left(\varepsilon^{2}\right) .
$$

This approximation for the nonlinear source distribution $f(\xi)$ is expressed by a linear contribution (inverting (3.17)) composed with $(S(\xi)$-dependent) iterates of linear objects: namely the linear $(S \equiv 0)$ Dirichlet-to-Neumann operator $D t N_{0}$ and again $\mathbf{P}^{-1}[\varphi]$. In the sequel we will confirm this interpretation for the $D t N_{0}$ operator, which is defined by the first sum in the expression for $\mathbf{R}_{S} \mathbf{P}^{-1}$. Thus we have approximated the calculation of the source distribution by straightforward Fourier transforms (namely FFTs) of smooth functions. We will now show how the nonlinear DtN operator can be approximated by straightforward compositions of the linear $D t N_{0}$ operator. This is achieved by using the approximation given in (3.21).

4. Evolution equations for weakly nonlinear surface gravity waves. In the context of nonlinear surface gravity waves, the Neumann data is needed at the second FS condition. Writing the norm (squared) of the velocity in terms of its tangential and normal components, implies that we need to find the $\xi$ and $\zeta$ derivatives as in

$$
\operatorname{DtN}[\varphi](\xi) \equiv \partial_{n} \phi(\xi, S)=\left[-\frac{S_{\xi}}{\left|\Gamma_{\xi}\right|} \phi_{\xi}+\frac{1}{\left|\Gamma_{\xi}\right|} \phi_{\zeta}\right]_{(\xi, S)} .
$$

First we differentiate (3.16) with respect to $\zeta$ and use (3.21) having $\zeta=S$. After a lengthy calculation we obtain the compact expression

$$
\phi_{\zeta}=D t N_{0}\left[\varphi-S D t N_{0}[\varphi]\right]-S \varphi_{\xi \xi}+O\left(\varepsilon^{2}\right)
$$

When $S \equiv 0, \zeta$ is the normal direction and (4.2) is exact, confirming the interpretation given to $D t N_{0}$. There are other ways of representing the $D t N_{0}[\varphi] \equiv \mathbf{T}\left[\varphi_{\xi}\right]$ :

$$
\mathbf{T}\left[\varphi_{\xi}\right]=-i \sum_{\kappa \neq 0} \tanh [2 \pi \kappa \sqrt{\beta}] \mathbf{F}_{\kappa}\left[\varphi_{\xi}\right] e^{2 \pi i \kappa \xi}
$$

Note that the dispersion relation appears as the symbol of the linear $D t N_{0}$ operator. For example in Berger and Milewski [3] a Fourier-type integral transform, also having the dispersion relation as its symbol, is used in studying surface gravity wave interaction and wave-turbulence. This also appears in the work by Craig and Sulem [5]. As mentioned in the Introduction, we point out that $\mathbf{T}$ is the periodic counterpart of the (singular) integral operator used by Matsuno [16] and also referred to in Craig and Sulem [5] as the Hilbert transform on the strip:

$$
\tilde{\mathbf{T}}\left[\varphi_{x}\right]=\frac{1}{2 \sqrt{\beta}} \int_{-\infty}^{\infty} \frac{\varphi_{x}\left(x^{\prime}\right)}{\sinh \left[\frac{\pi}{2 \sqrt{\beta}}\left(x-x^{\prime}\right)\right]} d x^{\prime} .
$$

Moreover it is important to notice that the operator acts on, slightly different, velocity components. Here we have a terrain-following $\left(\phi_{\xi}\right)$ velocity component along the FS, which clearly is not tangent neither to the undisturbed, nor the perturbed FS. On the other hand Matsuno uses the horizontal $\left(\phi_{x}\right)$ velocity component at the FS (see (18) in [16]). 
Recalling that the surface wave profile is denoted by $S(\xi, t) \equiv \varepsilon N(\xi, t)$ we put together equations (4.1) and (4.2), and use the operator $\mathbf{T}$, to write the asymptotic expression

$$
\left|\Gamma_{\xi}\right| \operatorname{DtN}[\varphi](\xi)=\mathbf{T}\left[\varphi_{\xi}\right]-\varepsilon\left\{\mathbf{T}\left[\left(N \mathbf{T}\left[\varphi_{\xi}\right]\right)_{\xi}\right]-\left(N \varphi_{\xi}\right)_{\xi}\right\}+O\left(\varepsilon^{2}\right) .
$$

Define $U \equiv \phi_{\xi}$ as the "horizontal" terrain-following velocity component. Then

$$
\varphi_{\xi}=U+\varepsilon N_{\xi} \mathbf{T}[U]+O\left(\varepsilon^{2}\right)
$$

and the normal derivative approximation reads as

$$
\left|\Gamma_{\xi}\right| \partial_{n} \phi=\mathbf{T}[U]-\varepsilon \mathbf{T}\left[N \mathbf{T}[U]_{\xi}\right]-\varepsilon(N U)_{\xi}+O\left(\varepsilon^{2}\right) .
$$

The kinematic condition, in terms of the normal derivative to $\zeta=\varepsilon N$, is written as

$$
N_{t}-\left|\Gamma_{\xi}\right| \partial_{n} \phi /\left(M(\xi)^{2} \sqrt{\beta}\right)=0 .
$$

Substituting (4.5) and using the fact that $\eta(x, t)=M(\xi) N(\xi, t)+O\left(\varepsilon^{2}\right)$ [23], we obtain a fully dispersive evolution equation for the wave elevation:

$$
\eta_{t}-\frac{1}{M \sqrt{\beta}}\left\{\mathbf{T}[U]+\varepsilon\left(\left(\frac{\eta U}{M}\right)_{\xi}+\mathbf{T}\left[\frac{\eta}{M} \mathbf{T}[U]_{\xi}\right]\right)\right\}=O\left(\varepsilon^{2}\right) .
$$

For the dynamic FS condition we start by taking its $\xi$-derivative. We should keep in mind that $\phi(\xi, S(\xi, t), t)=\varphi(\xi, t)$ is such that $d \phi / d t=\varphi_{t}=\phi_{t}+\phi_{\zeta} S_{t}$ and $\phi_{t}=\varphi_{t}-\phi_{\zeta} S_{t}$. Moreover $d\left(\phi_{t}\right) / d \xi=\varphi_{t \xi}-\phi_{\zeta \xi} S_{t}-\phi_{\zeta} S_{t \xi}$. Using approximations given above, together with (4.2), we have

$$
\begin{aligned}
\frac{d}{d \xi} \phi_{t} & =U_{t}+\varepsilon N_{\xi t} \mathbf{T}[U]+\varepsilon N_{\xi} \mathbf{T}[U]_{t}-\phi_{\zeta \xi} S_{t}-\phi_{\zeta} S_{t \xi}+ \\
+O\left(\varepsilon^{2}\right) & =U_{t}+\varepsilon N_{\xi} \mathbf{T}[U]_{t}-\varepsilon \mathbf{T}[U]_{\xi} N_{t}+O\left(\varepsilon^{2}\right) .
\end{aligned}
$$

Going back to the dynamic condition and using the fact that we are working with the velocity components $\phi_{\xi} \equiv U$ and $\phi_{\zeta}=\mathbf{T}[U]+O(\varepsilon)$, after some computation, we arrive at

$$
U_{t}+\eta_{\xi}+\varepsilon\left(\frac{1}{2 \sqrt{\beta}}\left(\left(\frac{U^{2}}{M^{2}}\right)_{\xi}+\left(\frac{1}{2 M^{2}}\right)_{\xi} \mathbf{T}[U]^{2}\right)-\left(\frac{\eta}{M}\right)_{\xi} \mathbf{T}\left[\eta_{\xi}\right]\right)=O\left(\varepsilon^{2}\right) .
$$

Dropping the $O\left(\varepsilon^{2}\right)$ terms in equations (4.6) and (4.7) we get a fully dispersive terrainfollowing Boussinesq system. For a flat bottom (i.e. $M(\xi) \equiv 1$ ) this system reduces to system (19)-(20) in Matsuno [16]. Also by expanding in $\beta$, for the weakly dispersive regime, we can recover the terrain-following Boussinesq system as in [23], when using the vertically averaged terrain-following velocity component (equation (5.12) in [23]). Moreover for $\beta>0.25$ it is known [6], from the linear potential theory, that the surface wave does not feel the bottom anymore. Therefore beyond a certain value of $\beta$ there is no point in generalizing Matsuno's formulation. We should also point out that linearizing system (4.6) and (4.7) (by setting $\varepsilon=0$ ) we have that

$$
\eta_{t}-\frac{1}{M \sqrt{\beta}}\{\mathbf{T}[U]=0,
$$




$$
U_{t}+\eta_{\xi}=0
$$

Eliminating $\eta$ from this system and performing a Fourier transform of the resulting second order equation (in the flat bottom case; $M \equiv 1$ ) clearly leads to the full dispersion relation. Hence we say that system (4.6)-(4.7) is fully dispersive.

Following Matsuno [16] system (4.6)-(4.7) can be transformed into a, variable coefficient, second order Boussinesq equation:

$$
\begin{aligned}
\sqrt{\beta} M \eta_{t t}+\mathbf{T}\left[\eta_{\xi}\right]+\varepsilon\{- & \frac{1}{2}\left(\frac{\left(\eta^{2}\right)_{\xi}}{M}\right)_{\xi}+\sqrt{\beta}\left(\frac{\eta_{t}}{M} \mathbf{T}^{-1}\left[M \eta_{t}\right]\right)_{\xi}+ \\
& \left.+\frac{\sqrt{\beta}}{2} \mathbf{T}\left[\eta_{t}^{2}+\mathbf{T}^{-1}\left[M \eta_{t}\right]^{2} \frac{1}{M^{2}}\right]_{\xi}-\mathbf{T}\left[\frac{\eta}{M} \mathbf{T}\left[\eta_{\xi}\right]\right]_{\xi}\right\}=0 .
\end{aligned}
$$

We point out that derivatives of the metric term $M(\xi)$ produce $O\left(\beta^{-1 / 2}\right)$ terms $[23$, 18]. For the flat bottom case $(M \equiv 1)$, using the identity $\mathbf{T}\left[\mathbf{T}^{-1}[f]^{2}\right]=\mathbf{T}\left[f^{2}\right]+2 \mathbf{T}[f] f$ given in [16], this equation reduces to Matsuno's main result (namely equation (22); c.f. definition of $\delta$ and $\kappa$ in [16] page 609). In the presence of the metric term the simplification of the equation, through the above identity, does not apply.

5. Numerical application. In Nachbin and Papanicolaou $[21,22,19]$ a Boundary Element Method (BEM), proposed by Salmon et al. [28], was adapted to the dimensionless linear potential theory equations. In particular to the regime where the topography is on a rapidly varying scale. We will now contrast our previous BEM formulation with the spectral method provided by the $D t N$ operator. This current presentation will be restricted to the linear regime over a flat bottom. This is equivalent to linearizing the scheme proposed by Craig and Sulem [5] which was restricted to flat bottoms. The fully nonlinear numerics in the presence of rapidly varying topographies will be presented elsewhere.

We consider the scaled linear $(\alpha=0)$ water wave equations:

$$
\begin{gathered}
\beta \phi_{x x}+\phi_{y y}=0 \quad \text { in } \Omega \\
\text { at } y=0 \quad\left\{\begin{array}{l}
\phi_{t}=-\eta \\
\eta_{t}=\frac{1}{\beta} \phi_{y}
\end{array}\right.
\end{gathered}
$$

with the "impermeability" condition along the topography given by

$$
\phi_{y}+\frac{\beta}{\gamma} h^{\prime}\left(\frac{x}{\gamma}\right) \phi_{x}=0 .
$$

The harmonic part of this problem can be recast in the form of a Boundary Integral Equation (BIE) through Green's third identity [19]:

$$
\sqrt{\beta} \theta_{\mathbf{P}} \phi(\mathbf{P})=\oint_{\partial \Omega}\left(\phi(\mathbf{Q}) \frac{\mathrm{d} \ln \rho}{\mathrm{d} \bar{n}}-\phi_{\bar{n}}(\mathbf{Q}) \ln \rho\right) \mathrm{d} \mathbf{Q},
$$

where $\left(x_{Q}, y_{Q}\right) \equiv \mathbf{Q} \in \partial \Omega, \rho^{2}=\left(x_{P}-x_{Q}\right)^{2}+\beta\left(y_{P}-y_{Q}\right)^{2}$ and

$$
\theta_{\mathbf{P}}= \begin{cases}\pi & \text { if } \mathbf{P} \in \text { smooth part of } \partial \Omega \\ \text { internal angle } & \text { if } \mathbf{P} \in \text { corner of } \partial \Omega \\ 2 \pi & \text { if } \mathbf{P} \in \Omega .\end{cases}
$$


The scaled normal derivative is defined as $\phi_{\bar{n}} \equiv\left(\beta \partial_{x} \phi, \partial_{y} \phi\right) \cdot \vec{n}$. The BIE was solved by using the Boundary Element Method. The functional relation between the Dirichlet and Neumann data is approximated by finite elements along the BIE [19]. This leads to numerical dispersion as will be indicated below. A linear system arises when using the collocation method for the residual of the boundary element approximation. This system is dense, nonsymmetric and ill-conditioned. In [21, 22, 19] accurate results where obtained by using double precision on a supercomputer (about 28 digits of precision).

One of the advantages of the BEM, as opposed to the Finite Difference Method, is that we may consider the normal derivative $\phi_{\bar{n}}$ as a nodal parameter, that is, as an unknown of the algebraic system of equations. This is an advantage for boundaries of complex shape. Note also that this enables the linear free surface equations to be interpreted as a system of Ordinary Differential Equations (ODE) in time:

$$
\begin{gathered}
\frac{d \phi}{d t}\left(x_{j}, t\right)=-\eta\left(x_{j}, t\right) \\
\frac{d \eta}{d t}\left(x_{j}, t\right)=\frac{1}{\beta} \mathcal{F}[\phi]\left(x_{j}, t\right)
\end{gathered}
$$

where $\mathcal{F}[\phi]$ indicates symbolically that $\phi_{y}$ has a functional dependence on $\phi$. Hence solving the BIE is like computing the vector field of these "ODEs" at each time step.

The evolution scheme in time is done through an implicit scheme. For the kinematic condition at $t^{n+1 / 2}$ we adopt the trapezoidal rule:

$$
\frac{\eta^{n+1}-\eta^{n}}{\Delta t}=\frac{1}{\beta} \frac{\phi_{y}^{n+1}+\phi_{y}^{n}}{2}+O\left(\Delta t^{2}\right)
$$

For the dynamic condition, also at $t^{n+1 / 2}$, we use

$$
\frac{\phi^{n+1}-\phi^{n}}{\Delta t}=-\left[\eta^{n}+\frac{\Delta t}{2 \beta}\left(\theta \phi_{y}^{n+1}+(1-\theta) \phi_{y}^{n}\right)\right]+O\left(\Delta t^{2}\right),
$$

where $\theta$ is the implicit scheme's parameter. In Nachbin and Papanicolaou [21] it was shown that numerical dispersion is kept to a minimum if $\theta=1 / 6$. This scheme was proposed by Liu and Liggett [14] with an empirical value of $\theta=0.17$, which is very close to $1 / 6$. We have only one numerical differentiation (in $\mathrm{t}$ ) and the local truncation error is $\mathrm{O}\left(\Delta t^{3}\right)$ on both free surface conditions.

Through the formulation presented in the present paper we may write the linear free surface conditions in the form

$$
\begin{gathered}
\frac{d \phi}{d t}\left(\xi_{j}, t\right)=-\eta\left(\xi_{j}, t\right) \\
\frac{d \eta}{d t}\left(\xi_{j}, t\right)=\frac{1}{\beta} D t N_{0}[\phi]\left(\xi_{j}, t\right),
\end{gathered}
$$

where $D t N_{0}$ denotes the linear Dirichlet-to-Neumann operator as before:

$$
\operatorname{DtN}_{0}[\varphi](\xi)=\sum_{\kappa \neq 0} 2 \pi \kappa \tanh (2 \pi \kappa \sqrt{\beta}) \mathbf{F}_{\kappa}[\varphi] e^{2 \pi i \kappa \xi} .
$$

This expression is exact. In contrast with the BEM this procedure has several advantages: 
(a) we only need to use two FFTs to calculate the normal derivative with great efficiency; in the BEM a dense nonsymmetric system had to be solved at each time step.

(b) the normal derivative of the potential is calculated with spectral accuracy and, for a well resolved surface wave, numerical dispersion is absent.

(c) as a consequence of the previous comment we use an explicit $(\theta=0)$ evolution scheme along the free surface without observing any kind of numerical phase lag.

(d) the use of a curvilinear coordinate system in the presence of highly corrugated boundaries improves numerical stability, namely acting as a preconditioner $[18,24]$.

The following numerical experiments corroborate with the comments above.

We consider a Gaussian pulse for the velocity potential's initial profile:

$$
\varphi(\xi)=e^{-\left(\xi-\xi_{0}\right)^{2} / \sigma^{2}},
$$

where the parameter $\sigma$ controls the pulse's width and therefore how broadband its Fourier content is. For the other initial condition along the FS, let $\phi_{t}(\xi, 0,0) \equiv$ $\psi(\xi)$ and we choose $\widehat{\psi}(k)=-i \omega \widehat{\varphi}(k)$ so that the initial wave is rightgoing. The corresponding free surface elevation is therefore the derivative of a Gaussian.

We consider four levels of dispersion $(\beta=0.01,0.1,1$, e 10) and compare the numerical evolution with the exact solution. We take (5.1) with $\sigma=\sqrt{0.3}$ together with the following discretization parameters: $\Delta \xi=0.0625$, for a total of $J=1024$ nodes in space; $\Delta t=0.01$ for a total of $N=4500$ steps in time. As mentioned all experiments where done with the explicit scheme $(\theta=0)$ presented above.

The numerical experiments are presented in Figs. 5.1 and 5.2, where the solid line represents the numerical solution and the dots the exact values. The agreement is very good. In particular as we increase the dispersion level the Airy-like oscillatory behavior is captured very accurately.

We are in the process of extending the numerical scheme for the nonlinear regime and comparing the potential theory results with several reduced (Boussinesq-type) models that we have formulated. Muñoz Grajales and Nachbin $[17,18]$ have thoroughly tested a Boussinesq solver for the terrain-following Boussinesq system [23]. Currently this scheme is being adapted to approximate a family of Boussinesq models [25], as for example the one presented by Quintero and Muñoz Grajales [26]. All these models are different Padé approximations to the full dispersion relation

$$
\omega^{2}=\frac{k}{\sqrt{\beta}} \tanh [k \sqrt{\beta}] .
$$

For example the Boussinesq model in $[17,18]$ is such that

$$
\omega^{2}=\frac{k^{2}}{1+\frac{1}{3} \beta k^{2}} .
$$

This is equivalent to the regularized Korteweg-de Vries equation, also known as the BBM model [2]. Of particular interest, we have observed that in the presence of a rapidly varying disordered topography, small differences on how the full dispersion relation is truncated affect the dynamics of the wave's rapidly fluctuating components. Detailed nonlinear $(D t N)$ numerics together with comparisons among a family of weakly dispersive Boussinesq-type systems are currently under study, in the regime of long waves interacting with a disordered topography. These results should appear in the near future. 

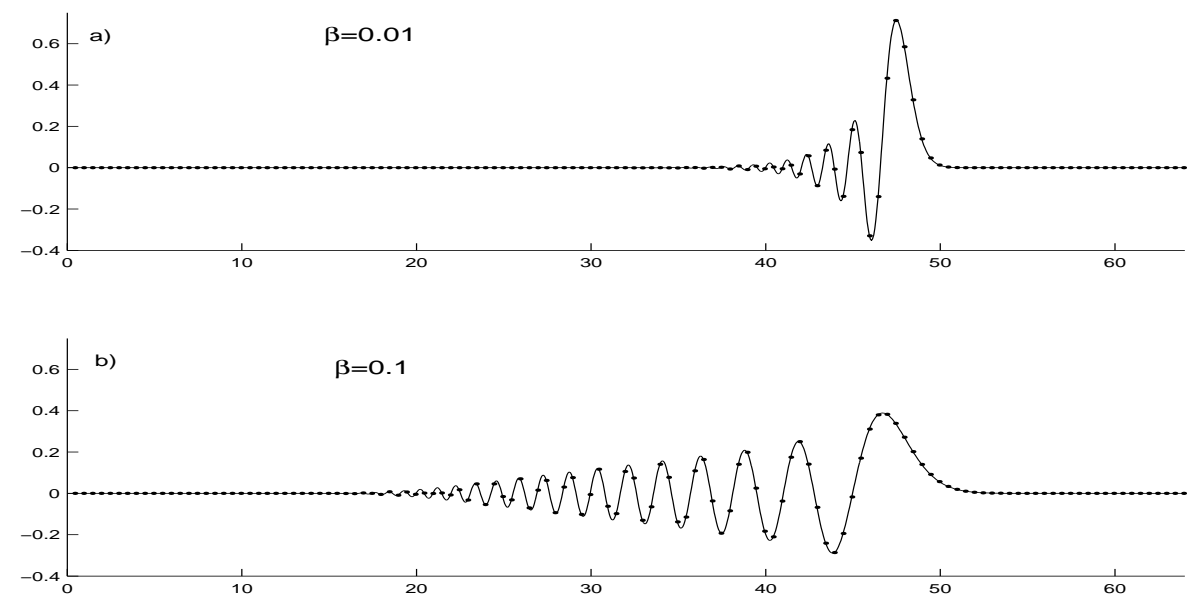

FIG. 5.1. Snapshot for a propagating velocity potential: two weakly dispersive regimes.
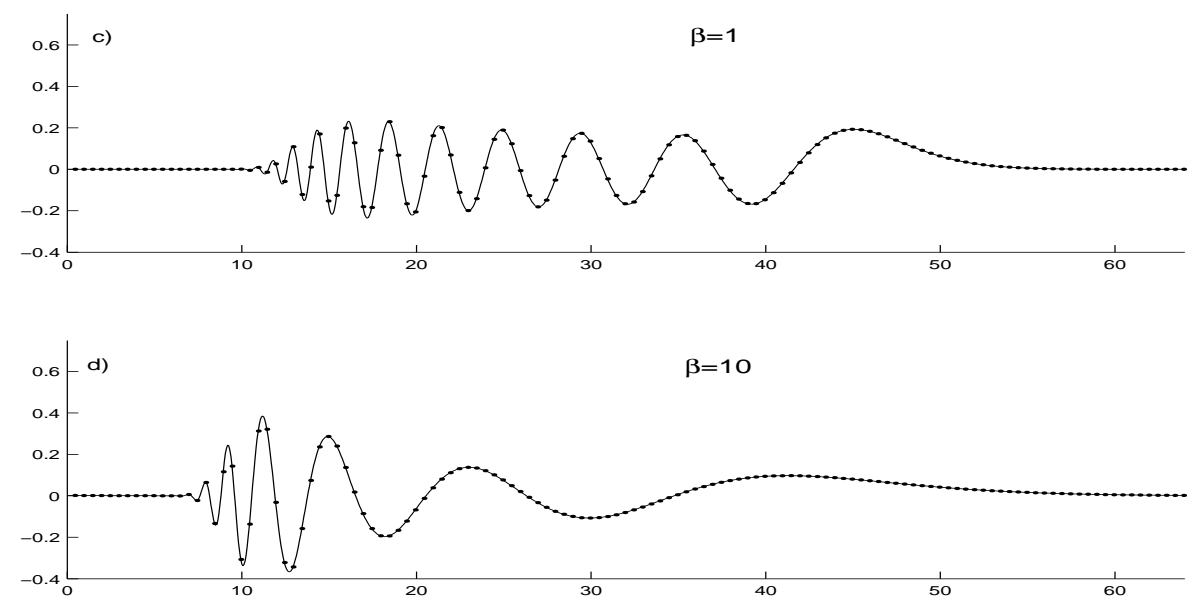

FIG. 5.2. Snapshot for a propagating velocity potential: dispersion fully developed.

6. Conclusions. We have generalized Matsuno's [16] Boussinesq-type equation for a general class of topography profiles as well as the terrain-following Boussinesq system [23] to being fully dispersive. The formulation is such that an efficient (FFT based) numerical scheme is readily available. In future work we expect to study the interaction of fully dispersive surface water waves over randomly varying topographies as in $[17,18,12,10,11]$. We also expect to explore higher dimensions where the conformal mapping does not apply. But an intermediate situation is the one where, for example, we have ridge like formations in the coastal region. In this case the topography is nearly one-dimensional but the wave (for example) can be generated as a point source, hence leading to a two-dimensional surface elevation.

The authors thank Prof. R. Kraenkel, (IFT/UNESP, São Paulo) for calling our attention to Matsuno's work [16]. 


\section{REFERENCES}

[1] W. ARtiles AND A. NAChBin, Nonlinear evolution of surface gravity waves over highly variable depth, Phys. Rev. Lett., Vol. 93, No. 23, 234501 (2004).

[2] T.B. Benjamin, J.L. Bona And J.J. Mahony, Model equations for long waves in nonlinear dispersive systems, Phil. Trans. Roy. Soc., A272 (1972), pp. 47-78.

[3] K.M. Berger and P.A. Milewski, Simulation of wave interactions and turbulence in onedimensional water waves, SIAM J. Appl. Math., 63:4 (2003), pp. 1121-1140.

[4] J.G.B. Byatт-Sмith, An integral equation for unsteady surface waves and a comment on the Boussinesq equation, J. Fluid Mech., 49 (1971), pp. 625-633.

[5] W. Craig and C. Sulem, Numerical simulation of gravity waves, J. Comput. Phys., 108 (1993), pp. $73-83$.

[6] R. Dean and R.A. Dalrymple, Water Waves Mechanics for Engineers and Scientists, World Scientific, 3rd. ed., 1993.

[7] T. DRISCOLL, http://www.math.udel.edu/ driscoll/software.

[8] T. Driscoll and L.N. Trefethen, Schwarz-Christoffel Mapping, Cambridge Univ. Press, 2002.

[9] J.P. Fouque And A. NAChBIn. Time-reversed refocusing of surface water waves, SIAM Multiscale Modeling and Simulation, 1:4 (2003), pp. 609-629.

[10] J.P. Fouque, J. Garnier and A. Nachbin. Time reversal for dispersive waves in random media, SIAM J. Appl. Math., 64:5 (2004), pp. 1810-1838.

[11] J.P. Fouque, J. Garnier and A. Nachbin, Shock structure due to stochastic forcing and the time reversal of nonlinear waves, Physica D, 195 (2004), pp. 324-346.

[12] J.P. Fouque, J. Garnier, J.C. Muñoz and A. Nachbin, Time reversing solitary waves, Phys. Rev. Lett., 92:9 (2004), 094502-1.

[13] P. Guidotti, A first-kind boundary integral formulation for the Laplace Dirichlet-to-Neumann map in 2D, J. Comput. Phy., 190 (2003), pp. 325-345.

[14] P.L.-F. LiU AND J.A. LigGetT, Boundary element formulations and solutions for some nonlinear water wave problems, Developments in Boundary Element Methods -2, Chap. 3, eds. P.K. Banerjee and R.P. Shaw (1984), Elsevier Applied Science Publishers.

[15] J.B. KelleR, Shallow-water theory for arbitray slopes of the bottom, J. Fluid Mech., 489 (2003), pp. 345-348.

[16] Y. Matsuno, Nonlinear evolution of surface gravity waves on fluid of finite depth, Phys. Rev. Lett., 69:4 (1992), p. 609.

[17] J.C. Muñoz Grajales and A. Nachbin, Dispersive wave attenuation due to orographic forcing, SIAM J. Appl. Math., 64:3 (2004), pp. 977-1001.

[18] J.C. MuÑoz GRAJAles AND A. NAChBIn. Stiff microscale forcing and solitary wave refocusing, SIAM Multiscale Model. Simul., 3:3 (2005), pp. 680-705.

[19] A. Nachbin, Modelling of Water Waves in Shallow Channels, Computational Mechanics Publications, Southampton, U.K., 1993.

[20] A. NAchBin, The localization length of randomly scattered water waves, J. Fluid Mech., 296 (1995), pp. 353-372.

[21] A. Nachbin and G.C. Papanicolaou, Boundary element method for the long-time water wave propagation over rapidly varying bottom topography, Intern. J. Num. Meth. in Fluids, 14 (1992), pp. 1347-1365.

[22] A. Nachbin And G.C. PapanicolaOu, Water waves in shallow channels of rapidly varying depth, J. Fluid Mech., 241 (1992), pp. 311-332.

[23] A. NachBin, A terrain-following Boussinesq system, SIAM J. Appl. Math., 63:3 (2003), pp. 905-922.

[24] D.P. Nicholls and F. Reitich, Stability of high-order pertubative methods for the computation of Dirichlet-Neumann operators, J. Comput. Phys., 170 (2001), pp. 276-298.

[25] O. NwoGu, Alternative form of Boussinesq equations for nearshore wave propagation, J. Waterway, Port, Coastal and Ocean Engineering, 119 (1993), pp. 618-638.

[26] J.R. Quintero And J.C. MuÑOz, Existence and uniqueness for a Boussinesq system with a disordered forcing, Meth. Appl. of Analysis, 11:1 (2004), pp. 15-32.

[27] R.R. Rosales And G.C. Papanicolaou, Gravity waves in a channel with a rough bottom, Studies in Appl. Math., 68 (1983), pp. 89-102.

[28] J.R. Salmon, P.L.-F. LiU And J.A. LigGett, Integral equation method for linear water waves, J. Hydraulic Division, ASCE, 106 (1980), pp. 1995-2010.

[29] M.E. TAYlor, Partial Differential Equations I: Basic Theory, Springer Verlag, 1996.

[30] G.B. Whitham Linear and Nonlinear Waves, John Wiley, (1974).

[31] V.E. Zakharov, A.I. Dyachenko and O.A. Vasilyev, New method for numerical simulation 
of a nonstationary potential flow of incompressible fluid with a free surface, Euro. J. Mech. B/Fluids, 21 (2002), pp. 283-291. 